Einführung 


\title{
Ziel und Anlage der Untersuchung
}

\author{
Ulrich Becker
}

I. Die Ausgangssituation

II. Ziel der Untersuchung und Fragestellungen

1. Feststellung von Prinzipien

2. Ausgangspunkt: Zur Rechtsstellung von Beamten

3. Die Bedeutung allgemeiner Reformen

III. Methodik und Ansatzpunkte des Vergleichs

1. Personaler Anknüpfungspunkt: Beamte

2. Sachlicher Anknüpfungspunkt: Alterssicherung

3. Rechtsordnungsinterne Vergleiche

IV. Zur Auswahl der Rechtsordnungen

1. Nationale Rechtsordnungen

2. Völker- und supranationale Rechtsordnungen

V. Aufbau der Untersuchung und der einzelnen Berichte

1. Vorverständnis und zuvor gesammelte Erfahrungen

2. Die den Berichten zugrundegelegten Fragen 


\section{Die Ausgangssituation}

1. Soziale Sicherungssysteme haben in vielen Ländern ein drittes Zeitalter erreicht. Nach der Errichtung und dem Ausbau erfahren sie in den letzten Jahren zum Teil grundlegende Reformen. ${ }^{1}$ Das läßt sich an der Entwicklung der gesetzlichen Rentenversicherung in Deutschland zeigen: Diente diese im 19. Jahrhundert noch vorrangig als Invaliditätssicherung auf dem Niveau der Armutsvermeidung, ${ }^{2}$ so entwickelte sie sich schrittweise zu einem Alterssicherungssystem, das seit der großen Rentenrefom 1957 eine Teilhabe am gesellschaftlichen Wohlstand sicherstellt. ${ }^{3}$ Dem weiteren Ausbau in den 1970er Jahren und der Ermöglichung der Vorruhestandspolitik in den 1980er Jahren folgte schon 1989 ein erster spürbarer Einschnitt. ${ }^{4}$ Mit der Einführung steuerlicher Subventionen für eine ersetzende private Zusatzsicherung zu Beginn des neuen Jahrtausends wurde und wird das Niveau der umlagefinanzierten Rente abgesenkt. ${ }^{5}$ Die zweite Säule, die bei funktionaler Betrachtung aus geförderter Privatversicherung und betrieblicher Vorsorge besteht, ${ }^{6}$ gewinnt an Bedeutung. Das alles heißt zwar nicht, daß die gesetzliche Rentenversicherung, die große integrative Erfolge sowohl nach Ende des Zweiten Weltkriegs als auch zur Bewältigung der Folgen der Wiedervereinigung 7 aufweisen kann, nicht auch in Zukunft in Deutschland der wichtigste Baustein der Alterssicherung bleiben wird - zumal kapitalgedeckte Systeme mit eigenen Unsicherheiten verbunden sind. ${ }^{8}$ Es gibt kein Allheilmittel, um die Folgen der demographischen Entwicklungen für die soziale Sicherung zu bewältigen. Eine Verschiebung zwischen den ein-

1 Vgl. zu den Reformen im Vergleich: Reinhard, Demographischer Wandel und Alterssicherung, Rentenpolitik in neun europäischen Ländern und in den USA im Vergleich, 2001; Feldstein/Siebert (eds.), Social Security Pension Reform in Europe, 2002; DRV (Hrsg.), Rentenversicherung im internationalen Vergleich, 2003; Holzmann/Orenstein/Rutkowski (eds.), Pension Reform in Europe: Process and Progress, 2003; Hughes/Stewart (Hrsg.), Reforming Pensions in Europe, 2004; Maltby/De Vroom/Mirabile/Overbye (eds.), Ageing and the Transition to Retirement, 2004; $\mathrm{Na}$ tali/Rhodes, The 'New Politics' of the Bismarckian Welfare State: Pension Reforms in Continental Europe, EUI Working Paper SPS No. 2004/10; Bonoli/Shinkawa (eds.), Ageing and Pension Reform Around the World, 2005; Fultz (ed.), Pension reform in the Baltic States, 2006; Barr, Reforming pensions, 2008.

2 Zur Geschichte Stolleis, Geschichte des Sozialrechts in Deutschland, 2003, S. 84 ff.

3 Vgl. nur Hockerts, Sozialpolitische Entscheidungen im Nachkriegsdeutschland, 1980, S. 320 ff.

4 Durch Umstellung der Rentenanpassung mit dem Rentenreformgesetz 1992 v. 18.12.1989 (BGB1. 1989 I, S. 2261).

5 Gesetz zur Ergänzung des Gesetzes zur Reform der gesetzlichen Rentenversicherung und zur Förderung eines kapitalgedeckten Altersvorsorgevermögens (AVmEG) v. 21.3.2001 (BGBl. 2001 I, S. 403).

6 Dazu Becker, Private und betriebliche Altersvorsorge zwischen Sicherheit und Selbstverantwortung JZ 2004, S. 846 ff.

7 Vgl. Ritter, Der Preis der Einheit. Die deutsche Wiedervereinigung und die Krise des Sozialstaats, 2. Aufl. 2007.

8 Vgl. Barr, Reforming pensions: Myths, truths and policy choices, ISSR 55 (2002), S. 3 ff. 
zelnen, zusammenwirkenden Bestandteilen der Alterssicherung 9 und damit eine breitere Verteilung der Unsicherheit auf verschieden aufgebaute Säulen ist aber nicht zu übersehen.

Die angedeuteten Entwicklungen sind keineswegs auf Deutschland beschränkt. Zumindest in allen industrialisierten Staaten, die - wenn auch mit Niveauunterschieden über ausgebaute soziale Sicherungssysteme verfügen, lassen sich gemeinsame Reformtrends feststellen: ${ }^{10}$ Verteilung der Alterssicherung auf mehrere und unterschiedlich finanzierte Säulen, Stärkung des Äquivalenzprinzips in der Standard- oder Regelsicherung und, wenn auch mit Unterschieden, Heraufsetzung des Regelrentenalters. Diese Trends werden innerhalb der Europäischen Union durch die sog. Methode der offenen Koordinierung ${ }^{11}$ verstärkt und aufeinander abgestimmt.

2. Die kurz zusammengefaßten Entwicklungen betreffen die allgemeine Alterssicherung, auf die sich viele Untersuchungen in den vergangenen Jahren konzentriert haben. Wie aber steht es mit der Alterssicherung von Beamten? Nicht ohne Grund darf man insofern mit einigen Besonderheiten rechnen. Zunächst sprechen dafür die historischen Wurzeln. Denn die Versorgung von Beamten im Alter dürfte das älteste staatliche soziale Sicherungssystem darstellen: Im 18. und zu Beginn des 19. Jahrhunderts entstanden entsprechende Versorgungssysteme. ${ }^{12}$ Hintergrund dessen ist der Aufbau einer

9 Zur Komplexität Zacher, Ziele der Alterssicherung und Formen ihrer Verwirklichung, in: ders. (Hrsg.), Alterssicherung im Rechtsvergleich, 1991, S. 25, $31 \mathrm{ff}$.

10 Vgl. dazu nur Becker, Alterssicherung im internationalen Vergleich, in: ders./Kaufmann/v. Maydell/Schmähl/Zacher (Hrsg.), Alterssicherung in Deutschland, Festschrift für Franz Ruland zum 65. Geburtstag, 2007, S. 575 ff.

11 Dazu allgemein: Die OMK nahm ihren Ausgangspunkt im „Weißbuch über Wachstum, Wettbewerbsfähigkeit und Beschäftigung", das die Kommission im Jahr 1993 unter ihrem damaligen Vorsitzenden Jaques Delors veröffentlichte, KOM(93) 700 endg. Sie wurde zwischenzeitlich ,gestrafft“, vgl. Kommission der Europäischen Gemeinschaften: Mitteilung für die Frühjahrstagung des Europäischen Rates. Zusammenarbeit für Wachstum und Arbeitsplätze. Ein Neubeginn für die Strategie von Lissabon, $\operatorname{KOM(2005)} 24$ endg., und ist eng verknüpft mit der so genannten Lissabon-Strategie, vgl. die erneuerte Sozialagenda, $\operatorname{KOM(2008)} 412$ endg. Vorschlag zur weiteren Verbesserung der OMK durch KOM(2008) 418 endg. Zur Alterssicherung: Gemeinsamer Bericht des Rates und der Kommission über angemessene und nachhaltige Renten, 7165/03. Grundsätzlich positive Bewertung von Ruland, Offene Methode der Koordinierung im Bereich der Alterssicherung in der Europäischen Union - Quo Vadis?, in: DRV (Hrsg.), Offene Koordinierung im Bereich der Alterssicherung - Quo Vadis?, 2003, S. 7 f.

12 Vgl. Wunder, Editorial, in: Pensionssysteme im öffentlichen Dienst in Westeuropa (19./20. Jh.), JEV Bd. 12, 2000, S. IX: „Belohnungen für Beamte durch den Herrscher gab es schon in der Frühen Neuzeit. Aber erst in der Aufklärung und besonders im Umbruch der Französischen Revolution und unter Napoleons Hegemonie über Europa wurde die Altersfürsorge im öffentlichen Dienst zu einem normierten System, das sich von den willkürlichen Einzelentscheidungen des Monarchen löste und die Versorgung nach objektiven Kriterien - Dienstalter und Karrierestufe - teilweise gesetzlich, d.h. als Rechtsanspruch des Beamten, festlegte." Als erste gesetzliche Grundlage auf deutschem Boden wird die bayerische „Hauptlandespragmatik über die Dienstverhältnisse der Staatsdiener vorzüglich in Beziehung auf ihren Stand und Gehalt" vom 1. Januar 1805 genannt, in der 
funktionierenden und rechtlich geordneten Verwaltung, in deren Folge der Staat zugunsten der Amtswalter und damit der Beamten eine besondere Fürsorgepflicht übernahm. Vom Beamten durfte Loyalität erwartet werden. Er erhielt dafür eine unkündbare Stellung und eine lebenslange Versorgung. Damit ist eine Verbindung angesprochen, die bis heute für die Ausgestaltung der Alterssicherung von entscheidender Bedeutung sein kann: Jedenfalls wenn und soweit eine besondere Rechtsbeziehung zwischen Staat und Beamten existiert, spricht einiges dafür, daß auch die Alterssicherung der Beamten durch Besonderheiten geprägt bleibt.

\section{Ziel der Untersuchung und Fragestellungen}

\section{Feststellung von Prinzipien}

Ziel der vorliegenden Untersuchung ist es, allgemeine Prinzipien der Alterssicherung von Beamten herauszuarbeiten. Von Interesse sind dabei denkbare Grundsätze, die den deutschen Lesern bekannt erscheinen, aber nicht aus dem bestehenden deutschen Recht, sondern dem vorstehend erwähnten, zumindest potentiellen funktionalen Zusammenhang zwischen der Rechtsstellung des Beamten und seiner Alterssicherung abgeleitet werden können. Gemeint sind damit das Alimentationsprinzip im Sinne einer umfassenden Verantwortung des Staates für die Unterhaltssicherung im Alter und das Prinzip einer amtsangemessenen Versorgung im Sinne einer Niveausicherung, die an dem zuletzt erreichten Amt ausgerichtet ist.

Allgemeinheit der Prinzipien meint in dieser Studie ein Zweites, nämlich die Feststellung eines internationalen Standards. Hier soll nicht nur berichtet werden, was in nationalen Rechtsordnungen und innerhalb von internationalen Organisationen gilt, sondern es soll vergleichend herausgearbeitet werden, ob und inwieweit von eigenen Prinzipien der Alterssicherung von Beamten gesprochen werden kann.

\section{Ausgangspunkt: Zur Rechtsstellung von Beamten}

Die Suche nach den genannten Prinzipien setzt aber zunächst die Beantwortung der Frage voraus, ob überhaupt eigene Alterssicherungssysteme für Beamte existieren bzw. ob die Alterssicherung von Beamten Besonderheiten aufweist. Das mag dem deutschen Leser, der mit der Beamtenversorgung in Deutschland vertraut ist, als überflüssig er-

„zum ersten Mal Konzeptionen eines modernen Beamtentums Gesetz geworden [sind], die zwei zentrale Postulate umschlossen: die Unkündbarkeit der Beamten und die Garantie einer Versorgung für sie“, so Zacher, Die Versorgung der Beamten, Richter und Soldaten, 1984, S. 7. 
scheinen, ist aber angesichts der Tatsache, daß einige Länder universelle Sicherungssysteme eingerichtet haben, ein erster und unerläßlicher Schritt. Hingewiesen sei nur auf die skandinavischen Länder, die bereits in der ersten Hälfte des 20. Jahrhunderts einheitliche Sicherungssysteme geschaffen haben, in welche die Beamten wie andere Erwerbstätige einbezogen worden sind. ${ }^{13}$ Andere Länder haben die Beamtensicherung als beitragsbezogene Vorsorge angelegt oder externalisiert, ${ }^{14}$ d.h. aus dem Beschäftigungsverhältnis selbst gelöst und als beitragsfinanzierte Vorsorge einem eigenständigen Versicherungsträger anvertraut. ${ }^{15}$

Aber es ist noch ein Schritt weiter zu gehen. Denn es kann keineswegs als selbstverständlich angesehen werden, daß in allen Rechtsordnungen eine Gruppe von Beschäftigten existiert, die in einem besonderen Verhältnis zum Staat steht, deren Aufgaben also eine besondere Loyalität zum Staat oder anderen öffentlich-rechtlichen Einrichtungen fordern, der dann eine besondere Fürsorge dieser Einrichtungen oder des Staats gegenübersteht, die sich wiederum in Besonderheiten der Alterssicherung niederschlägt. Zwar müssen für den Staat, der das Gewaltmonopol beansprucht, natürliche Personen handeln. Diesen Organwaltern ist mit ihrem Amt dann auch die Ausübung von Hoheitsgewalt anvertraut. Und erst die Herausbildung eines Beamtentums im Sinne gut ausgebildeter und nur dem Gemeinwohl verpflichteter Amtswalter hat den modernen Staat ermöglicht, der effektiv reguliert und seinen Bürgern die zur Daseinsvorsorge dienenden Einrichtungen effektiv zur Verfügung stellt. Ohne dieses sich in Europa langsam entwickelnde „arbeitsteilige Fachbeamtentum“ hätte, so Max Weber, die „Gefahr furchtbarer Korruption und gemeinen Banausentums“ bestanden und wäre die „rein technische Leistung des Staatsapparats" bedroht worden. 16 Jedoch zeigt die weitere Entwicklung, daß nicht nur fraglich ist, welcher Personenkreis im wahrsten Sinne des Wortes staatstragende Funktionen ausübt, sondern auch, daß es verschiedene Wege

13 In Schweden wurde für bestimmte Staatsdiener (Richter, Militär, Pastoren) 1878 ein Pensionssystem eingeführt; das ersten Volksrentengesetz folgte erst 1913. Im Jahr 1916 beschloß der Reichstag, daß eine Sicherung in bestehenden „Sondersystemen“ nicht zu einer Befreiung von der allgemeinen Versicherungspflicht führen sollte, weil eine Volksrente nur „solidarisch“ wirksam sein könne; vgl. Köhler, Funktion und rechtliche Ausgestaltung der Zusatzversicherungssysteme bei der Reform der Alterssicherungssysteme. Das Beispiel Schweden. in: Schlachter/Becker/Igl (Hrsg.), Funktion und rechtliche Ausgestaltung zusätzlicher Alterssicherungssysteme, Funktion und rechtliche Ausgestaltung zusätzlicher Alterssicherung Reformen in der Schweiz, den Niederlanden, Großbritannien, Schweden und Deutschland mit ihren internationalen und gemeinschaftsrechtlichen Bezügen, 2005, S. $85,93 \mathrm{f}$.

14 Vgl. zu Frankreich und Italien nur Becker, Staat und autonome Träger im Sozialleistungsrecht, Rechtsvergleichende Untersuchung der Risikoabsicherungssysteme in Deutschland, Frankreich, Italien und Großbritannien, 1996, S. 236 f. und $341 \mathrm{ff}$.

15 In diesem Zusammenhang zum Begriff der Externalisierung Zacher, Grundtypen des Sozialrechts, in: Festschrift für Zeidler, Bd. 1, 1987, S. 571 ff.

16 Weber, Politik als Beruf (1919), Gesamtausgabe Bd. 17 (hrsg. Mommsen/Schluchter), 1992, S. 175 f. 
gibt, diese besondere Funktionalität zu schützen. ${ }^{17}$ Eine gewisse „Normalisierung“ hat dabei auch das Europäische Gemeinschaftsrecht mit sich gebracht. Danach sind nicht nur Beamte als Arbeitnehmer anzusehen. ${ }^{18}$ Die für die Ausübung einer „Beschäftigung in der öffentlichen Verwaltung" vorgesehene Ausnahme von der Arbeitnehmerfreizügigkeit (Art. 45 Abs. 4 AEUV = ex-Art. 39 Abs. 4 EGV) ist nach der Rechtsprechung des Europäischen Gerichtshofs funktional zu verstehen und restriktiv auszulegen. ${ }^{19}$ Dementsprechend wird der Kreis der Personen, für die eine besondere Loyalität im Sinne der eigenen Staatsangehörigkeit gefordert werden darf, durch das Europäische Gemeinschaftsrecht eng gezogen. Daß deshalb das gesamte Beamtentum oder einzelne Sonderstellungen von Beamten überflüssig geworden wären, ist damit natürlich nicht gesagt. Daß aber entsprechende Sonderstellungen zunehmend unter Rechtfertigungsdruck geraten, ist nicht zu übersehen.

\section{Die Bedeutung allgemeiner Reformen}

Wo aber auch in der Alterssicherung besondere Gestaltungen für Beamte feststellbar sind, bleibt zu prüfen, welche Auswirkungen die eingangs genannten Reformtrends auf entsprechende Systeme haben. Denn zumindest einige Reformansätze dürften mit möglichen beamtenspezifischen Prinzipien der Alterssicherung konfligieren. Insbesondere der Ausbau der Beitragsäquivalenz im Sinne einer Berücksichtigung der lebenslang gezahlten Beiträge ${ }^{20}$ löst die Rentenleistung von der zuletzt erreichten beruflichen Stellung und widerspricht damit - zumindest noch stärker als zuvor - auch dem Grundsatz einer amtsangemessenen Versorgung (vgl. vorstehend II.1.). Sollten entsprechende Entwicklungen zu beobachten sein, stellt sich die Frage, inwieweit diese als Anlaß genom-

17 Auch deshalb wird heute das Beamtenrecht in Deutschland vielfach als überholt empfunden; vgl. dazu und dem Verhältnis zwischen Beamtenversorgung und Sozialversicherung Hase, Rentenversicherung und Beamtenversorgung, in: Becker u.a., Alterssicherung in Deutschland (Fußn. 10), S. $495 \mathrm{ff}$.

18 Eine bemerkenswerte Entscheidung des EuGH ist die in der Rs. Vougioukas, in der die Zusammenrechnung von Versicherungszeiten auf Beamte ausgedehnt worden ist, und zwar unter unmittelbarem Rückgriff auf die primärrechtliche Verpflichtung zur Einführung einer Koordinierung und das Diskriminierungsverbot, weil die bestehenden Koordinierungsverordnungen für Beamte nicht galten, EuGH v. 22.11.1995, Rs. C-443/93, Slg. 1995, I-4033. Zurückhaltender zur Invaliditätssicherung EuGH v. 20.4.1999, Rs. C-360/97 (Nijhuis), Slg. 1999, I-1919, und zwar deshalb, weil es angesichts unterschiedlicher Sicherungssysteme insofern erst einer gesetzgeberischen Festlegung bedurfte.

19 Zur autonomen Auslegung des Begriffs der öffentlichen Verwaltung, zum funktionellen Verständnis und den strengen Voraussetzungen nur EuGH v. 2.7.1996, Rs. C-473/93 (Kommission/Luxemburg), Slg. 1996, I-3207, Rdnr. 26 f., 33.

20 So etwa in Frankreich, Italien und Österreich, in deren Rentenversicherungssystemen zuvor nur eine beschränkte Zahl von „besten“ Jahren als Grundlage für die Rentenberechnung herangezogen wurde, vgl. nur Becker, in: ders. u.a., Alterssicherung in Deutschland (Fußn. 10), S. 575, 591 f., 595 . 
men werden, um auf eine weitere Gleichbehandlung zu drängen. Allerdings dürfte das schon deshalb eine sehr untergeordnete Rolle spielen, weil die meisten Länder ohnehin nur in beschränktem Umfang Sonderregelungen für die Alterssicherung von Beamten vorsehen, die Herstellung von Gleichheit deshalb in diesem Zusammenhang keine besondere Brisanz entfaltet.

\section{Methodik und Ansatzpunkte des Vergleichs}

\section{Personaler Anknüpfungspunkt: Beamte}

Die hier durchgeführte Untersuchung verbindet funktionale mit institutionellen Fragen. Das ist an sich für den Sozialrechtsvergleich keine Besonderheit. Im Gegenteil ist diese Verschränkung im Sozialleistungsrecht die Regel. Denn welche Einrichtungen für die soziale Sicherheit geschaffen werden, ist nicht funktionsbedingt, sondern unterliegt weitgehend politischer Dezision. Erst in der Folge ergeben sich dann daraus bestimmte rechtliche Fragen, etwa nach der Bedeutung der Vorsorge oder der Einbindung von dritten Leistungserbringern. ${ }^{21}$ Das hindert nicht den Vergleich. Es beschränkt aber unter Umständen, wenn nämlich einem funktionalen Ansatz gefolgt wird, die mit dem Vergleich zu gewinnenden, rechtlich relevanten Schlußfolgerungen.

Im folgenden geht es um die Verbindung der Alterssicherung zu bestimmten Tätigkeiten für den Staat oder andere öffentlich-rechtliche Einrichtungen und um die Auswirkungen der gesellschaftlichen Entwicklungen auf diese mögliche Verbindung. Das sind funktionale Fragestellungen. Zur Vereinfachung werden diejenigen Personen, die besondere Funktionen für den Staat ausüben, als Beamte bezeichnet bzw. es wird, etwas allgemeiner, von einer Beschäftigung im „öffentlichen Dienst“ ausgegangen. Das ist deshalb ein gangbarer Weg, weil alle Vergleichsstaaten die genannten Begriffe kennen. Hätte man stattdessen eng an eine bestimmte Funktion anknüpfen wollen, dann hätte es nahegelegen, sich etwa auf die Alterssicherung von Polizisten oder vielleicht sogar von Verkehrspolizisten zu konzentrieren. Stattdessen werden hier alle Personen einbezogen, die nach jeweils eigenem Verständnis und damit auch nach jeweils eigener Definition als Beamte, civil servants etc. bzw. als Beschäftigte in einem öffentlichen Dienst anzusehen sind. Insofern wird die funktionelle Betrachtung um eine institutionelle angereichert. Das hat einen guten Grund. Denn es sollten nicht nur eng umschriebene Tätigkeitsbereiche erfaßt werden. Ziel ist es vielmehr, allgemein zu klären, ob und inwieweit in den verschiedenen Rechtsordnungen an die Ausübung bestimmter Tätigkeiten Be-

21 Vgl. dazu näher Becker, Rechtsvergleich und Rechtsdogmatik im Sozialrecht, in: ders. (Hrsg.), Rechtsvergleich und Rechtsdogmatik im Sozialrecht I, 2010, S. 11 ff. 
sonderheiten geknüpft werden. Dafür aber spielt es nur eine untergeordnete Rolle, für welche einzelnen Tätigkeiten vom Vorliegen solcher Besonderheiten ausgegangen wird.

\section{Sachlicher Anknüpfungspunkt: Alterssicherung}

Auch der zweite Anknüpfungspunkt, der Begriff der Alterssicherung, bedarf einer Erläuterung. Das Alter zählt zu den „Risiken“, die nach internationalen ${ }^{22}$ und supranationalen Vorschriften ${ }^{23}$ durch Sozialleistungen abgesichert werden, ohne daß es dazu hier näherer Ausführungen bedürfte. ${ }^{24}$ Enger hingegen ist der Begriff der Beamtenversorgung. In Deutschland werden unter Versorgungssystemen internalisierende Sicherungssysteme verstanden, weshalb neben der Alters- und Invaliditätssicherung der Beamten auch die betriebliche Altersvorsorge als Versorgung bezeichnet wird. Gemeint ist also eine bestimmte institutionelle Ausgestaltung, auf die ein Vergleich gerade nicht beschränkt werden darf, wenn er umfassend die Vorkehrungen zur Alterssicherung von Beamten zum Gegenstand haben soll.

Die Untersuchung konzentriert sich ferner auf das Risiko des Alters. Sie blendet andere Risiken, insbesondere das der Invalidität bzw. der Erwerbsunfähigkeit aus, und zwar ganz unabhängig von der Frage, ob die einzelnen nationalen Sicherungssysteme mehrere Risiken zusammenfassen oder nicht.

Andererseits ist es erforderlich, nicht nur die staatliche Regel- oder Standardsicherung in den Blick zu nehmen. In den meisten Ländern wird die Aufgabe der Alterssicherung auf mehrere Säulen oder Schichten verteilt, und zwar in zunehmendem Maße. ${ }^{25}$ Würde man dieses Zusammenspiel außer acht lassen, bliebe das Bild unvollständig. Das gilt in besonderem Maße für die Sicherung der Beamten: Die internalisierende Sicherung in Deutschland kommt ohne ergänzende Systeme aus. Sie ist dennoch von den Leistungen her gesehen bifunktional, d.h. sie umfaßt sowohl die Standard- oder Regelsicherung als auch eine betriebliche Sicherung. ${ }^{26}$ In anderen Ländern, die keine interna-

22 Hingewiesen sei auf Art. 25 ff. („Leistungen bei Alter“) des IAO-Abkommens Nr. 102 über Mindestnormen der sozialen Sicherheit.

23 Insofern genügt der Hinweis auf die Koordinierungsverordnungen (Art. 44 ff. VO 1408/71: „Alter und Tod (Renten)“, bzw. Art. 50 ff. VO 883/2004: „Alters- und Hinterbliebenenrenten“). Zu diesen Verordnungen Becker, in: Schwarze u.a. (Hrsg.), EU-Kommentar, 2. Aufl. 2009, Art. 42 EGV, Rdnr. $12 \mathrm{ff}$.

24 Vgl. auch zur Anknüpfung für Rechtsvergleiche Zacher, Vorfragen zu den Methoden der Sozialrechtsvergleichung, in: ders. (Hrsg.), Methodische Probleme des Sozialrechtsvergleichs, 1977, S. $21,42,46 \mathrm{ff}$.

25 Dazu nur Becker, Generalbericht unter besonderer Berücksichtigung der Rechtslage in Deutschland, in: Schlachter/Becker/Igl, Funktion und rechtliche Ausgestaltung zusätzlicher Alterssicherungssysteme (Fußn. 13), S. 107 ff.

26 Grundl. Ruland, Möglichkeiten und Grenzen einer Annäherung der Beamtenversorgung an die gesetztliche Rentenversicherung, Anlageband B zum Gutachten der Sachverständigenkommission Alterssicherungssysteme, 1983, Rdnr. 56. 
lisierenden Systeme (mehr) kennen, wird sich auch die Alterssicherung der Beamten auf mehrere Säulen verteilen. Für die Vergleichbarkeit ist es deshalb erforderlich, ergänzende und insbesondere betriebliche Alterssicherungssysteme in die Betrachtung einzubeziehen. ${ }^{27}$

\section{Rechtsordnungsinterne Vergleiche}

Schließlich erfordert das Untersuchungsziel, auch innerhalb der einzelnen Landesberichte Vergleiche anzustellen. Dieser rechtsordnungsinterne Vergleich betrifft die Alterssicherung der Beamten einerseits und die der übrigen Erwerbstätigen andererseits. In vielen Ländern, nämlich denen, in denen Beamte in die allgemeinen gesetzlichen Systeme einbezogen sind, kann er sich auf die Sicherungssysteme der zweiten Säule beschränken.

Die durch einen rechtsordnungsinternen Vergleich gewonnenen Erkenntnisse sind die Grundlage, um beamtenspezifische Prinzipien der Alterssicherung abzuleiten. Auch insofern gilt, daß erst die vergleichende Betrachtung die relevanten Erkenntnisse ermöglicht: Erst durch sie werden mögliche Besonderheiten der Alterssicherung von Beamten sichtbar. Dieses induktive Vorgehen ermöglicht eine Abstraktion, das Lösen von der positiven Ausformung einzelner nationaler Sicherungssysteme und dementsprechend die Feststellung allgemeiner und übergreifender Grundsätze.

\section{Zur Auswahl der Rechtsordnungen}

\section{Nationale Rechtsordnungen}

Da der Vergleich dazu dient, die Grundsätze für die Alterssicherung von Beamten herauszuarbeiten, konnte auf einen möglichst breit angelegten Überblick verzichtet werden. Zwar spielt sicher auch die Frage eine Rolle, welche Ausgestaltungen dieser Alterssicherung wie oft anzutreffen ist und damit die Regel darstellt. Dieser quantitative Aspekt soll aber einem qualitativen hintangestellt werden, zumal einzelne Charakteristika von sozialen Sicherungssystemen angesichts deren Komplexität nur in ihrer Bedeu-

27 Was natürlich auch in ökonomischen Studien zu berücksichtigen wäre, vgl. aber die Studie des DIW zum Altersvermögen vom Januar 2010, abrufbar unter http://www.diw.de/documents/publikationen/73/diw_01.c.345836.de/10-3.pdf. 
tung eingeschätzt werden können, wenn die Einbettung in das Gesamtsystem Berücksichtigung findet. 28

Die Auswahl der Vergleichsländer ließ sich vor diesem Hintergrund von mehreren Überlegungen leiten. Von geringster Bedeutung war eine Kategorisierung europäischer Länder nach Grundformen sozialer Sicherung. ${ }^{29}$ Denn solche Kategorisierungen müssen, im übrigen nach mittlerweile wohl einhelliger Ansicht, als zu grob angesehen werden. ${ }^{30}$ Auch machen Reformtrends nicht an den Grenzen der verschiedenen Kategorien halt. Im Hinblick auf das Untersuchungsziel wurde die Auswahl deshalb vorrangig von zwei Fragen geleitet: Erstens davon, in welchen Ländern sich bestimmte Typen von Alterssicherungssystemen beispielhaft finden lassen. ${ }^{31}$ Zweitens davon, wo im internationalen Vergleich als besonders bemerkenswert einzustufende Reformmaßnahmen durchgeführt worden sind. Dazu kam ergänzend ein dritter Punkt, nämlich das Bemühen, innerhalb Europas eine weite regionale Verteilung der Vergleichsländer anzustreben. Einbezogen wurden deshalb neben nord- und zentraleuropäischen auch südeuropäische und osteuropäische Länder. Gerade die letztgenannten Länder fehlen, selbst 20 Jahre nach der Überwindung der politischen Blockbildung in Europa, noch in vielen Rechtsvergleichen, so daß auf die Schließung dieser Lücke besonderer Wert gelegt wurde.

In Konkretisierung der genannten allgemeinen Kriterien lassen sich die in den Vergleich einbezogenen Länder folgendermaßen schlagwortartig charakterisieren: Deutschland als Mutterland der Sozialversicherung verfügt über ein ausgeprägtes Sondersystem der Beamtenversorgung. Frankreich ist zwar ebenfalls ein Sozialversicherungsstaat mit einer besonderen Beamtensicherung, die aber nicht internalisierend angelegt ist. Das Vereinigte Königreich steht im Gegensatz zu den beiden zuerst genannten Staaten für eine universell angelegte soziale Sicherung, deren Niveau, zumindest soweit es die staatlichen Sicherungssysteme angeht, im Vergleich zu den beiden erstgenannten Ländern spürbar abgesenkt ist. Die drei skandinavischen Länder Dänemark, Norwegen und Schweden werden einbezogen, weil diese über ein traditionell eigenständiges Sicherungsmodell verfügen, das aber, wie insbesondere das Beispiel Schweden zeigt, von allgemeinen Reformtrends nicht verschont bleibt. Die Schweiz zeichnet sich dadurch aus, daß sie in der Alterssicherung schon seit langem über ein ausgeprägtes Mehrsäu-

28 Vgl. hingegen zu einem Versuch, anhand grober Kategorien und einer umfassenden Zählung zur Ableitung von Grundsätzen zu kommen, Mares, Social protection around the world, Comparative Political Studies, 2005, S. $623 \mathrm{ff}$.

29 Grundlegend Esping-Andersen, The Three Worlds of Welfare Capitalism, 1990; ders., Social Foundations of Postindustrial Economies, 1999.

$30 \mathrm{Vgl}$. etwa aus jüngerer Zeit Obinger/Castles/Leibfried, Introduction, in: dies. (eds.), Federalism and the Welfare State, 2005, S. 1 ff.

$31 \mathrm{Vgl}$. etwa zu den Grundstrukturen die Beiträge in Zacher, Alterssicherung im Rechtsvergleich (Fußn. 9), S. 115 ff.; VDR (Hrsg.), Rentenversicherung im internationalen Vergleich, DRVSchriften Bd. 15, 1999 und (als 2. Aufl.) DRV-Schriften Bd. 45, 2003. 
lensystem verfügt. ${ }^{32}$ Italien ähnelt in der Anlage dem französischen Modell, ist aber zugleich den südeuropäischen Staaten zuzurechnen, für die zuweilen eine eigene Kategorie des residualen Sozialstaats gebildet wird. Schließlich wurden mit Rußland, Slowenien und Tschechien drei osteuropäische Länder aufgenommen, die sich in verschiedenen Entwicklungsstadien befinden, zumal nur die beiden letztgenannten Mitgliedstaaten der Europäischen Union sind.

\section{Völker- und supranationale Rechtsordnungen}

Internationale Organisationen beschäftigten Personal, dessen Rechtsstellung durch besondere Statuten festgelegt wird. Diese Beschäftigten werden - weil und soweit sie mit der Erfüllung der besonderen öffentlichen Aufgaben ihrer Organisationen betraut sind - als internationale Beamte bezeichnet. ${ }^{33}$ Sie unterliegen einem besonderen Alterssicherungssystem, wie insgesamt ihre soziale Sicherung, auch um Unabhängigkeit und Loyalität sicherzustellen, den Internationalen Organisationen selbst obliegt.

Bei der Ausgestaltung der Alterssicherung verfolgen Internationale Organisationen zwei Ziele. Zum einen sollen für die gewöhnlich aus einer Vielzahl von Staaten stammenden internationalen Beamten weitgehend gleiche Regelungen der Dienstverhältnisse geschaffen werden. Damit kann die personelle Durchlässigkeit innerhalb der Organisationssysteme ermöglicht und ein Wettbewerb der Organisationen bei der Rekrutierung von Mitarbeitern vermieden werden. Zum anderen sollen die Alterssicherungssysteme der Organisationen so gestaltet sein, daß sie zwar dem aus dem Gedanken der Souveränität der Internationalen Organisationen folgenden Erfordernis der Befreiung von nationalen Sicherungssystem entsprechen, zugleich aber auch eine versorgungsrechtlich problemlose Rückkehr internationaler Beamter in die Entsendestaaten ermöglichen.

In diesem Spannungsfeld nehmen die Internationalen Organisationen die in den Mitgliedstaaten existierenden Prinzipien der Alterssicherung von Beamten auf. Sie sind aber nicht nur gehalten, eine Überversorgung zu vermeiden, sondern darüber hinausgehend auch ihre eigenen Sicherungssysteme entsprechend den allgemeinen Trends zu reformieren, ${ }^{34}$ nicht zuletzt um die Belastungen der Mitgliedstaaten, die im Ergebnis die Kosten der sozialen Sicherung tragen müssen, in einem angemessenen Rahmen zu halten. Angesichts dessen ist ein Vergleich der Alterssicherung in Internationalen Organisationen mit der von Beamten in den Mitgliedstaaten aufschlußreich. Mit dem Dienst-

32 Vgl. dazu nur Kaempfe, Die Systemfunktion privater Altersvorsorge im Gesamtsystem sozialer Alterssicherung, 2005.

33 Vgl. Seidl-Hohenveldern/Loibl, Das Recht der Internationalen Organisationen, 7. Aufl. 2000, Rdnr. 1042 ff.; vgl. auch Bornemann, Das Recht der Bediensteten internationaler Organisationen, 1964, S. 46.

34 Vgl. zu Reformen in einigen Organisationen ausführlich WEU, Document A/1934, Pension Schemes in the Coordinated Organisations, 2006, S. 7 ff. 
recht der Vereinten Nationen und der Versorgung der Beamten der Europäischen Union werden deshalb zwei Rechtsordnungen in die Untersuchung einbezogen, die von besonderer praktischer Bedeutung sind.

\section{Aufbau der Untersuchung und der einzelnen Berichte}

\section{Vorverständnis und zuvor gesammelte Erfahrungen}

Auch wenn sich der Rechtsvergleicher bei der Formulierung der zu untersuchenden Fragen von der eigenen Rechtsordnung lösen soll, ist es doch zumeist die Beschäftigung mit dieser Rechtsordnung, die ihm den Anstoß für eine vergleichende Untersuchung liefert. ${ }^{35}$ Das ist unvermeidbar und auch solange unschädlich, wie in Kenntnis dieses Umstands der Versuch unternommen wird, das positive Recht auf die von diesem zu beantwortenden Fragen des Lebens zurückzuführen. 36

Für die Alterssicherung der Beamten sind die mit der deutschen Rechtsordnung gesammelten Erfahrungen besonders aufschlußreich. Denn hier weist diese Sicherung nicht nur eine große Eigenständigkeit auf, sondern es sind auch schon deren tragende Prinzipien in früheren Studien herausgearbeitet worden. Insofern ist es sinnvoll, die entsprechenden Prinzipien als Umschreibungen für mögliche Besonderheiten der Alterssicherung von Beamten (vgl. oben, II.1.) auch bei der Untersuchung anderer Rechtsordnungen im Hinterkopf zu behalten (vgl. unten, V.2.(I.)) und zu prüfen, ob sie sich auch dort finden lassen. Deshalb wird im folgenden vor die einzelnen Berichte über nationale und internationale Rechtsordnungen ein grundlegender Beitrag gestellt. In diesem Beitrag werden die in Deutschland gesammelten Erfahrungen mit der Rechtsvergleichung zur Alterssicherung von Beamten zusammengefaßt und bewertet, und es wird zudem eine deutsche Besonderheit klargestellt: Nämlich die starke Prägung der deutschen Beamtenversorgung durch die Verfassung und die Rechtsprechung des Bundesverfassungsgerichts.

35 Vgl. dazu grundl. Esser, Möglichkeiten und Grenzen des dogmatischen Denkens im modernen Zivilrecht, AcP 172 (1972), S. 97, 103 ff., $110 \mathrm{ff}$.

36 Ein solcher Induktionsschluß ist zwar nicht logisch zwingend, aber deshalb keineswegs fehlerhaft, vgl. nur Canaris, Funktion, Struktur und Falsifikation juristischer Theorien, JZ 1993, S. 377, 383. 


\section{Die den Berichten zugrundegelegten Fragen}

(I.) Grundlegung

(1.) Bestehen im Vergleichsland Unterschiede zwischen Beschäftigten innerhalb des öffentlichen Dienstes, beispielsweise bei der Begründung des Beschäftigungsverhältnisses und dessen inhaltlicher Ausgestaltung?

(2.) Gibt es ein „Berufsbeamtentum“ als besonderen rechtlichen Status der Beamten? Sind im Rahmen von Beamtenverhältnissen verfassungsrechtliche Grundsätze zu beachten?

(3.) Erfordern die Besonderheiten der Beamtenstellung eine besondere soziale Sicherung der Beamten im Alter?

Hinweis: In Deutschland beispielsweise hat die Regelung und Fortentwicklung des öffentlichen Dienstes unter Berücksichtigung der hergebrachten Grundsätze des Berufsbeamtentums zu erfolgen (Art. 33 Abs. 5 Grundgesetz). Die Grundsätze des Berufsbeamtentums erfassen alle Prinzipien, die für die Institution des Berufsbeamtentums prägend sind. Dazu gehören die Prinzipien, Beamte lebenslang und angemessen zu alimentieren sowie deren Versorgung am zuletzt ausgeübten Amt auszurichten.

(II.) Ausgestaltung der Altersabsicherung von Beamten

(1.) Die soziale Absicherung von Beamten im Alter wird im Vergleichsland getrennt vom allgemeinen Alterssicherungssystem geregelt:

(a) Inwiefern unterscheidet sich die Alterssicherung von Beamten von der im allgemeinen System?

(b) Welche Leistungen sind vorgesehen?

(c) Welche Leistungsvoraussetzungen müssen vorliegen?

(d) Wie erfolgt die Leistungsbemessung, u.U. unter Berücksichtigung besonderer Grundsätze und eines bestimmten Sicherungsniveaus?

(e) Wie wird die Alterssicherung von Beamten finanziert und wer trägt die Finanzierungskosten?

Hinweis: Um die Besonderheiten der Alterssicherung für Beamten zu verdeutlichen, sollten die allgemeinen Alterssicherungssysteme im Vergleich dargestellt werden. Bestehen diese allgemeinen Alterssicherungssysteme aus mehreren Säulen, dann sollten die einzelnen Bestandteile und deren Zusammenspiel ebenso aufgezeigt werden.

(2.) Das Vergleichsland räumt Beamten keinen Sonderstatus ein bzw. die Altersabsicherung von Beamten wird nicht getrennt vom allgemeinen Alterssicherungssystem geregelt: 
(a) Welche obligatorischen oder freiwilligen Systeme zur sozialen Absicherung im Alter bestehen für Erwerbstätige?

(aa) Sind sie einbezogen in ein allgemeines Sicherungssystem, besteht beispielsweise eine allgemeine Sozialversicherung?

(bb) Gibt es Zusatzsysteme (etwa kommunal) für einzelne Berufe oder betriebliche Systeme für Angehörige einzelner Unternehmen?

(cc) Existieren private Altersvorsorgesysteme, die u.U. staatlich gefördert werden?

(b) Wie sind die Alterssicherungssysteme ausgestaltet?

(aa) Handelt es sich um beitragsdefinierte (defined contribution) oder um leistungsdefinierte (defined benefit) Systeme?

(bb) Welche Leistungen sind vorgesehen und wie erfolgt die Leistungsbemessung? Gibt es beispielsweise eine Mindestsicherung?

(cc) Wie erfolgt die Finanzierung und wer ist Träger der Alterssicherungssysteme?

Hinweis: Wenn die bestehenden Alterssicherungssysteme - seien es die Sonderregelungen zur Altersabsicherung der Beamten oder die allgemeinen Alterssicherungssysteme in den letzten Jahren reformiert worden sind, dann sind die Gründe für diese Reformen und die alte Rechtslage an den relevanten Stellen kurz darzustellen, um die erfolgten Änderungen kenntlich zu machen.

(III.) Auswertung

(1.) Bestehen im Bereich des öffentlichen Dienstes des Vergleichslandes besondere öffentlich-rechtliche Beschäftigungsverhältnisse in Form von Beamtenverhältnissen?

(2.) Stellt im rechtlichen System des Vergleichslandes ein Beamtenverhältnis besondere Anforderungen an die Absicherung der beamteten Bediensteten im Alter dar? Welche spezifischen Grundsätze müssen dabei beachtet werden?

(3.) Sind Auswirkungen der Reformen allgemeiner Alterssicherungssysteme auf die Beamtenversorgung zu verzeichnen? Bestehen u.U. Konflikte zwischen Reformen bzw. Reformbestrebungen und besonderen Regelungsprinzipien der Alterssicherung von Beamten? 


\title{
Erfahrungen des Rechtsvergleichs und verfassungsrechtliche Vorgaben in Deutschland
}

\author{
Peter A. Köhler
}

I. Zehn Jahre Rechtsvergleich: Die Arbeitsergebnisse der neuen „Fachgruppe“ der Gesellschaft für Rechtsvergleichung und der von der Bundesregierung eingesetzten „Studien-“ und „Fachkommission“"

1. Die Fachgruppe der Gesellschaft für Rechtsvergleich

2. Die Studienkommission für die Reform des öffentlichen Dienstrechts

3. Die Sachverständigenkommission Alterssicherung

II. Das Beamtentum in der Krise - krisenfest?

1. Der 39. Deutsche Juristentag 1951

2. Das Urteil des BVerfG zum Gesetz zu Art. 131 GG als Thema der Staatsrechtslehrertagung 1954 - Rückbesinnung auf die Staatsrechtslehrertagung 1931

III. Beamtentum, aktuelle Reformversuche und das Bundesverfassungsgericht

1. Beamtenrecht versus Beamtentum

2. „beachten“" versus „,berücksichtigen“ - „Modifizierung“ versus „Veränderung“

3. „Traditionsbildender Zeitraum“ versus Regelungsbedürfnisse der Gegenwart 